\title{
MAGNETIC PRODUCTION OF EXTRAGALACTIC JETS
}

\author{
M. CAMENZIND \\ Landessternwarte Königstuhl \\ D-6900 Heidelberg 1 \\ Federal Republic of Germany
}

\begin{abstract}
Relativistic jets are formed by plasma injected into the rapidly rotating magnetosphere of accretion disks. The accretion disk generates a dipolar or a quadrupolar magnetosphere which also immerses the black hole. We present self-consistent solutions for the structure of this magnetosphere based on the GradSchlüter-Shafranov equation for the poloidal magnetic flux. The resulting supermagnetosonic jets consist of a nested family of magnetic surfaces filled with normal plasma flowing along the rotational axis of the central object.
\end{abstract}

\section{Introduction}

Magnetic fields are essential ingredients for modern theories of active galactic nuclei. Magnetic fields exist everywhere in a galaxy as shown by the interstellar matter of our own Galaxy and by many radio observations of jet sources in elliptical galaxies. It is now commonly assumed that the jets in quasars and extragalactic radio sources are generated by a compact supermassive object (of a mass $M \geq 10^{6} M_{\odot}$ ) in the nucleus of the host galaxy. Relativists believe that a supermassive black hole is the prime candidate for the compact object, but alternative theories of gravity also allow for non-collapsed rapidly rotating supermassive objects. Accretion onto these supermassive objects then ultimately provides the energy dissipated into radiation and jets.

\section{Large Scale Magnetic Fields in Accretion Disks}

We consider a rotating black hole surrounded by a magnetized accretion disk. The origin of the magnetic fields in accretion disks must be due to the existence of seed fields in the disks which extend to the larger scales of a few parsecs to a few hundreds of parsecs in galaxies. These larger disks and rings represent the reservoir for gas and magnetic flux which are ultimately accreted onto the central black hole.

As the disk's plasma accretes in the potential of the central mass, magnetic field lines are convected inwards, amplified and finally deposited on the horizon of the black hole. Since the disk's plasma can never be treated in the infinite conductivity limit, the question of the structure of the magnetic field in the inner disk around 
the black hole (say for $R \leq 100 R_{G}$ ) will depend on the outer boundary conditions for the magnetic fields, on the expression for the conductivity in the disk plasma as well as on possible dynamo effects in accretion disks.

According to present concepts used in dynamo theories, helical turbulence, which arises due to the combined action of rotation and convection, will generate a magnetic field in a differentially rotating system. In particular, in radiation pressure dominated disks thermal convection will develop. Large-scale magnetic fields will affect the convective turbulence and make it anisotropic. This results then in a magnetic helicity $\alpha_{B}$. The total helicity obtained from such a model is given by $\alpha_{B} \simeq \Omega L_{z} \sqrt{L_{z} / H}$ (Lominadze and Chagelishvili, 1983). $L_{z}$ is the characteristic size of a turbulent cell, $L_{z} \leq H$.

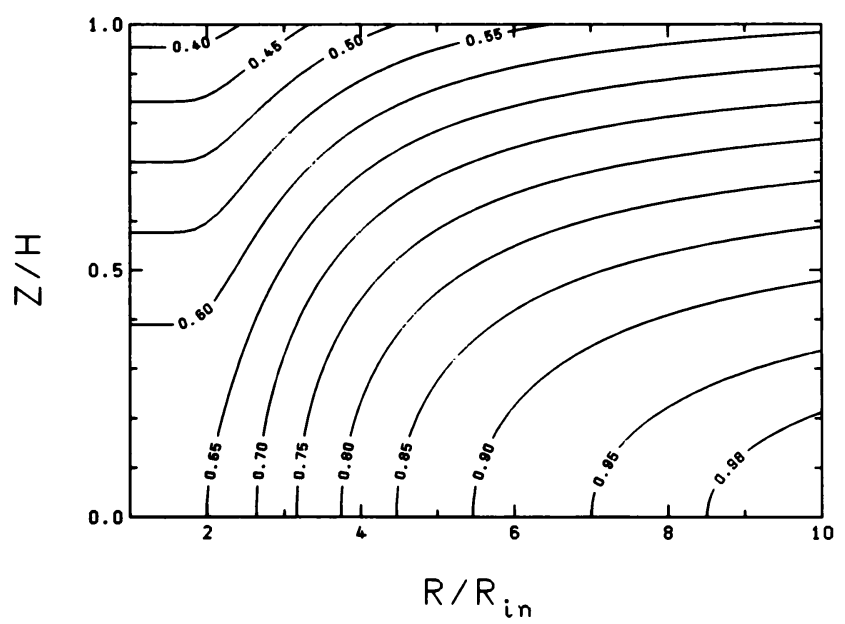

Fig. 1. Dipolar field structure in turbulent accretion disks around black holes $\left(\alpha_{B} \equiv 0\right)$. The turbulent diffusivity $\eta_{T}$ vanishes near the horizon $R_{\text {in }}$ and far from the hole. $H$ is the vertical height of the disk.

The evolution of the magnetic fields in geometrically thin accretion disks is quite similar to the processes found in galactic disks for the time evolution of the magnetic flux $\Psi$ and the toroidal magnetic field $B_{\phi}$

$$
\begin{aligned}
\frac{\partial \Psi}{\partial t}+u_{R} \frac{\partial \Psi}{\partial R} & =\eta_{T} \frac{\partial^{2} \Psi}{\partial z^{2}}+R \alpha_{B} B_{\phi}, \\
\frac{\partial B_{\phi}}{\partial t}+\frac{\partial}{\partial R}\left(u_{R} B_{\phi}\right) & =\eta_{T} \frac{\partial^{2} B_{\phi}}{\partial z^{2}}+\frac{3}{2} \frac{\Omega}{R} \frac{\partial \Psi}{\partial z}+4 \pi \frac{\partial \eta_{T}}{\partial R} j_{z} .
\end{aligned}
$$

Since $\alpha_{B}<\Omega H$, the generation of magnetic fields in the disks is given in the $\alpha \Omega$ approximation, and $B_{z} / B_{\phi} \simeq O(H / R)$. The toroidal component of the magnetic field is therefore substantially intensified after a few revolutions and grows to the maximal value $B_{\phi}^{2} \simeq 8 \pi P_{\text {disk }}, B_{\phi} \simeq 100 \mathrm{kG} \dot{M}_{26}^{1 / 2} M_{H, 8}^{-1}\left(R_{\text {in }} / R\right)^{5 / 4}$. 


\section{Jets as Dipolar Plasma Flows from Rotating Magnetospheres}

It is well known that the rapid rotation of magnetized neutron stars has dramatic effects on the physics of the magnetosphere (for a recent review, see Camenzind $1989 \mathrm{~b})$. Similar effects must occur when the rapid rotation of supermassive objects drives currents of the order of $10^{18}$ Ampère (see also Benford, 1987). Plasma is injected into the rotating magnetosphere from a corona above the disk. The flow of this plasma is essentially determined by the strength of the magnetic field at the surface of the disk and the form of the magnetic surfaces built up by the rapid rotation. In the force-free limit, the stream function $\Psi(R, z)$ is a solution of the non-linear relativistic Grad-Schlüter-Shafranov equation

$$
\nabla \cdot\left\{\frac{\alpha}{R^{2}}\left(1-\frac{R^{2}\left(\Omega^{F}-\omega\right)}{\alpha^{2}}\right) \nabla \Psi\right\}=-\frac{\left(\Omega^{F}-\omega\right)}{\alpha} \frac{d \Omega^{F}}{d \Psi}(\nabla \Psi)^{2}-\frac{I(\Psi)}{\alpha R^{2}} \frac{d I}{d \Psi} .
$$

$R$ is the cylindrical radius, $\Omega^{F}(\Psi)$ the rotation of the field lines, $\omega$ the frame dragging effect and $\alpha$ the redshift factor of the gravitational background. This equation is a generalization of the pulsar equation (Macdonald \& Thorne, 1982). The inertia of the plasma produces additional terms in the current of equ. (3) (Camenzind, $1987,1989 \mathrm{a}) . I(\Psi)$ is a measure for the poloidal current circulating along the magnetic surfaces, $R B_{\phi}=-I(\Psi)$. This equation has to be solved for disk boundary conditions following from our discussion in Sect. 2.

In Fig. 2 we show a particular solution of this equation for $\Omega^{F} \simeq \Omega_{H} / 2(\alpha \equiv 1$, $\omega \equiv 0)$. The magnetic surfaces covering the black hole must stay within the light cylinder $R_{L}=c / \Omega^{F}$, since along these field lines plasma can only accrete onto the hole. The field lines emanating from the accretion disk are loaded with plasma, cross the light cylinder and are finally collimated outside the light cylinder. The typical jet radius is $R_{\text {jet }} \simeq(10-100) R_{L}$.

The poloidal velocity $u_{p}$ of the plasma streaming along a given rotating magnetic surface follows from the solutions of the hot wind equation (Camenzind, 1987; Camenzind \& Endler, 1989)

$$
\sum_{n=0}^{16} A_{n}\left(\frac{R}{R_{L}}, E, L, \frac{P_{\mathrm{in}}}{n_{\mathrm{in}} m c^{2}} ; \frac{\Phi}{\sigma_{\mathrm{in}}} ; g_{t t}, g_{t \phi}, g_{\phi \phi}\right) u_{p}^{n / 3}=0 .
$$

$\Phi$ is the normalized flux function for the magnetic surface. The only parameter which essentially determines the asymptotic velocity is Michel's magnetization parameter $\sigma_{\text {in }} \simeq \Psi_{\text {jet }}^{2} / 2 \dot{M} c R_{L}^{2}$ with $\sigma_{\text {in }} \simeq 10$ for quasar jets. Due to the particular form of the magnetic surfaces around the light cylinder, most of the magnetic energy is converted into kinetic energy: $u_{p \text {,jet }} \simeq 0.7 \sigma_{\text {in }}$. Only a small fraction of the magnetic energy is carried away in the form of a Poynting flux (Camenzind \& Endler, 1989). Jet solutions start with small velocities near the slow magnetosonic point, cross the Alfvén point inside and the fast magnetosonic point outside the light cylinder. Since inertia is included in our treatment, the light cylinder is no singular point. 


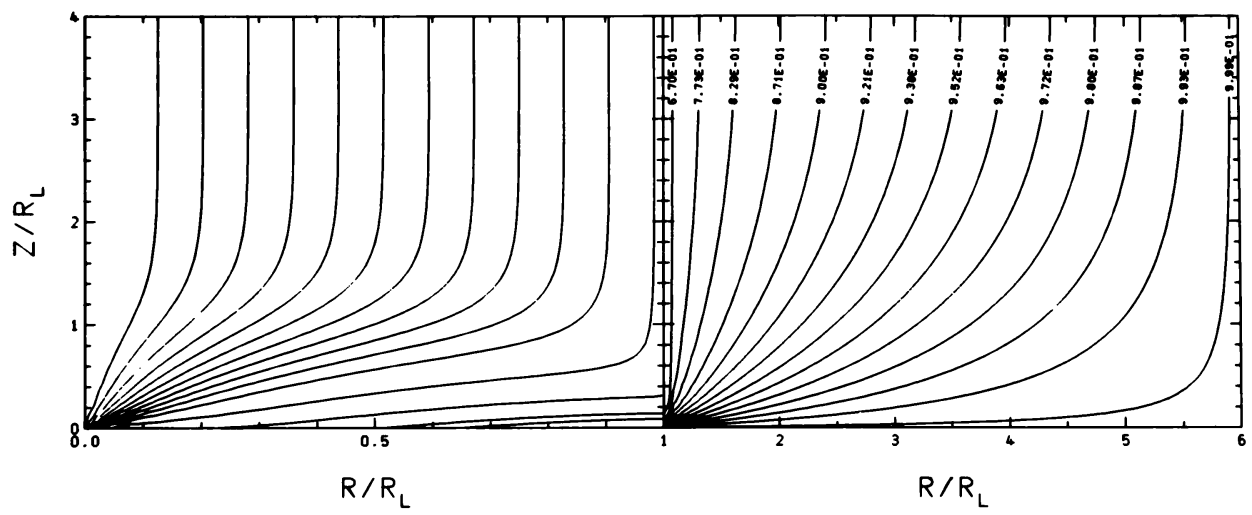

Fig. 2. Poloidal field lines of the rotating magnetosphere around the accretion disk. The magnetic flux which covers the black hole is confined to the region inside the light cylinder, while the flux emanating from the accretion disk crosses the light cylinder $R_{L}$.

Jets formed in rapidly rotating magnetospheres consist therefore of a family of nested magnetic surfaces including a current system flowing along these surfaces and closed at locations along the jet where the conductivity in the jet plasma is lowered by turbulent processes. For strongly magnetized jets, this mainly occurs in the hot spot region, where the supermagnetosonic plasma flow is decelerated by the ambient medium (Appl \& Camenzind, 1988; Lesch et al., 1989).

\section{References}

Appl, S., Camenzind, M.: 1988, Astron. Astrophys. 206, 258

Benford, G.: 1987, in Astrophysical Jets and their Engines, ed. W. Kundt, Reidel (Dordrecht)

Camenzind, M.: 1987, Astron. Astrophys. 184, 341

Camenzind, M.: 1989a, in Accretion Disks and Magnetic Fields in Astrophysics, ed. G. Belvedere, Kluwer (Dordrecht), p. 129

Camenzind, M.: 1989b, in Neutron Stars: their Birth, Evolution, Radiation and Winds, ed. W. Kundt, Kluwer (Dordrecht), in press

Camenzind, M., Endler, M.: 1989, submitted to Astron. Astrophys.

Lesch, H., Appl, S., Camenzind, M.: 1989, submitted to Astron. Astrophys.

Lominadze, J.G., Chagelishvili, G.D.: 1983, in Nonlinear and Turbulent Processes in Physics, ed. R.Z. Sagdeev, Harwood (London), p. 367

Macdonald, D.A., Thorne, Kip S.: 1982, Mon. Not. Roy. Astron. Soc. 198, 345 
INOUE: Recently we made mm-VLBI observations at $100 \mathrm{GHz}$ which gave $\sim 50 \mu$ arcsec resolution. The preliminary result on $3 \mathrm{C273}$ shows some blobs within 1 pc from the core. Unlike the well collimated jets from several pc to kpc scale, these blobs are not collimated, so that these should be collimated at around $1 \mathrm{pc}$ from the core. Hence my question: Is it possible to collimate these blobs at $\sim 1$ pc?

CAMENZIND: In 3C273, the jet is formed on the scale of one light year (the central mass $\simeq 10^{9} \mathrm{M}_{\odot}, R_{\mathrm{L}} \simeq 10$ Schwarzschild radii, and a jet radius $\simeq 100 R_{L}$ ). The blobs you observe are located within this scale and are produced most probably by resistive instabilities at the outer edge of the jet, where the toroidal field is strongest (this is well-known from plasma confinement in a Tokamak). The emission knots observed on the scale of a few parsecs represent the evolved structures and are more distributed over the jet. The relativistic particles generated in the inner-parsec knots are diffused over the whole jet and carried with the underlying plasma. This is the reason for the link between jet velocity and velocity of moving knots.

TSINGANOS: What is the energy equation you are using? Are there other solutions besides the one going through the three critical points?

CAMENZIND: We use a polytropic equation of state. The total energy is determined by the position of the fast magnetosonic point. The whole phase-plot $\left(u_{p}, R\right)$ is covered by solutions. The jet solution is required to pass through all 3 critical points. The position of the fast magnetosonic point is however strongly dependent on the structure of the magnetic surface. 